\title{
Hybridization of plasmonic antenna and cavity modes: Extreme optics of nanoparticle-on-mirror nanogaps
}

\author{
C. Tserkezis, ${ }^{1,}{ }^{*}$ R. Esteban, ${ }^{1}$ D. O. Sigle, ${ }^{2}$ J. Mertens, ${ }^{2}$ L. O. Herrmann, ${ }^{2}$ J. J. Baumberg, ${ }^{2}$ and J. Aizpurua ${ }^{1, \dagger}$ \\ ${ }^{1}$ Centro de Física de Materiales (CFM) CSIC-UPV/EHU and Donostia International Physics Center (DIPC), \\ Paseo Manuel de Lardizabal 4, Donostia-San Sebastián 20018, Spain \\ ${ }^{2}$ Nanophotonics Centre, Cavendish Laboratory, Department of Physics, JJ Thomson Avenue, University of Cambridge, \\ Cambridge CB3 OHE, United Kingdom
}

(Received 12 March 2015; published 4 November 2015)

\begin{abstract}
The precise structural details of metallic nanogaps within optical antennae are found to dramatically modify the plasmonic response, producing a complex pattern of electromagnetic modes that can be directly observed in scattering experiments. We analyze this situation theoretically in the nanoparticle-on-mirror construct, which forms a plasmonic nanogap sensitive to even atomic-scale restructuring of nanoparticle morphology. We focus on the effect of nanoparticle faceting, which allows the formation of ultrathin cavities between the particle and the underlying metallic film in the nanoparticle-on-mirror geometry. Two different sets of modes are identified: longitudinal antenna modes, which are strongly radiative and excited for all facet width ranges, and transverse cavity modes produced at large facets and exhibiting extreme confinement. The interaction and hybridization of antenna and cavity modes is determined by their symmetry and the precise morphology of the nanogap edges. Understanding such complex optics from nanoparticle-on-mirror structures is important to elucidate a wide variety of emerging photochemical and optoelectronic processes.
\end{abstract}

DOI: 10.1103/PhysRevA.92.053811

PACS number(s): 42.60.Da, 73.20.Mf, 78.67.Pt

\section{INTRODUCTION}

The optical response of metallic nanostructures is mainly governed by the collective excitation of the conduction electrons, giving rise to surface charge density oscillations known as localized surface plasmons. Electromagnetically coupling plasmonic modes has attracted considerable interest because of the many possibilities for forming hybrid plasmonic modes with unusual functionalities [1,2]. A canonical example is the plasmonic nanogap, where coupled plasmonic modes confine and enhance optical fields, thus making this structure a very attractive building block for field-enhanced spectroscopy [3-6], photocatalysis [7], or active control in optoelectronics [8]. Recent advances in nanofabrication access sub-nm-scale optics using plasmonic gaps produced by appropriate molecular linkers [9-11], self-assembly [12], or state-of-the-art nanolithography $[13,14]$. A promising route towards controlling plasmonic gaps is provided by the nanoparticle-on-mirror (NPoM) geometry, where a metallic nanoparticle (NP) is brought to interact with its mirror image in an underlying metal film. Thin dielectric spacers between the two metallic interfaces are usually introduced to prevent conductive contact [15-18]. Large numbers of plasmonic nanogaps can thus be obtained in a controllable and reproducible manner. However, the exact geometry of the overlying particle strongly modifies the optical response of this system, making it a subtle optical monitor of atomic-scale modifications.

Here we present a detailed theoretical study of the effect of NP faceting on the optical response of the NPoM geometry, using it as a canonical construct that sustains processes involving reconstruction and chemical reconfiguration of limited

\footnotetext{
*Present address: Technical University of Denmark, Department of Photonics Engineering, Ørsteds Plads Building 343, 2800 Kgs. Lyngby, Denmark.

$\dagger$ aizpurua.ehu.es
}

nanoareas, difficult to access by any other characterization method [18]. We show that by introducing a flat facet at the bottom of the NP, where a nanogap between the NP and the complex spacer-film substrate is formed (as observed in experiments), and by gradually increasing the width of this facet, a set of transverse cavity modes is progressively excited in the gap [19-21]. These cavity modes can be efficiently coupled and hybridized with the standard longitudinal gap antenna modes. Understanding the conditions for excitation and interaction of these two sets of modes thus provides a crucial model to interpret morphological changes at the atomistic level.

\section{OPTICAL RESPONSE OF NPOM PLASMONIC SYSTEMS}

We consider a spherical gold NP of diameter $D=100 \mathrm{~nm}$, separated from a 70-nm-thick gold film (modeled here as a wide but finite gold disk) by a dielectric spacer with thickness $d=0.6 \mathrm{~nm}$. Such a spacer thickness, appropriate for example with two-dimensional monolayer materials such as $\mathrm{MoS}_{2}$, is close to the limit below which quantum effects become relevant $[22,23]$, but can be still considered large enough to allow an accurate description of the optical response of the system within classical electrodynamics (nonlocal corrections can be conveniently included when necessary $[15,24,25])$. The dielectric function of gold $\left(\varepsilon_{\mathrm{m}}\right)$ is taken from the literature [26], and for the spacer we use a constant permittivity $\varepsilon_{\mathrm{d}}=1.63$ [27]. We assume that the spherical NP is in contact with the underlying spacer via a facet of circular cross section of diameter $w$. The system is illuminated by a plane wave with wave vector $\mathbf{k}$ parallel to the NPoM axis ( $x$ axis) and electric field $\mathbf{E}$ with amplitude $E_{0}$ polarized along the NPoM axis normal to the substrate ( $z$ axis), as shown in Fig. 1(a). Extinction, scattering, and absorption cross sections $\left(\sigma_{\text {ext }}, \sigma_{\text {scat }}\right.$, and $\left.\sigma_{\text {abs}}\right)$, and corresponding near-field maps, are calculated using the boundary element method $[28,29]$. 
(a)
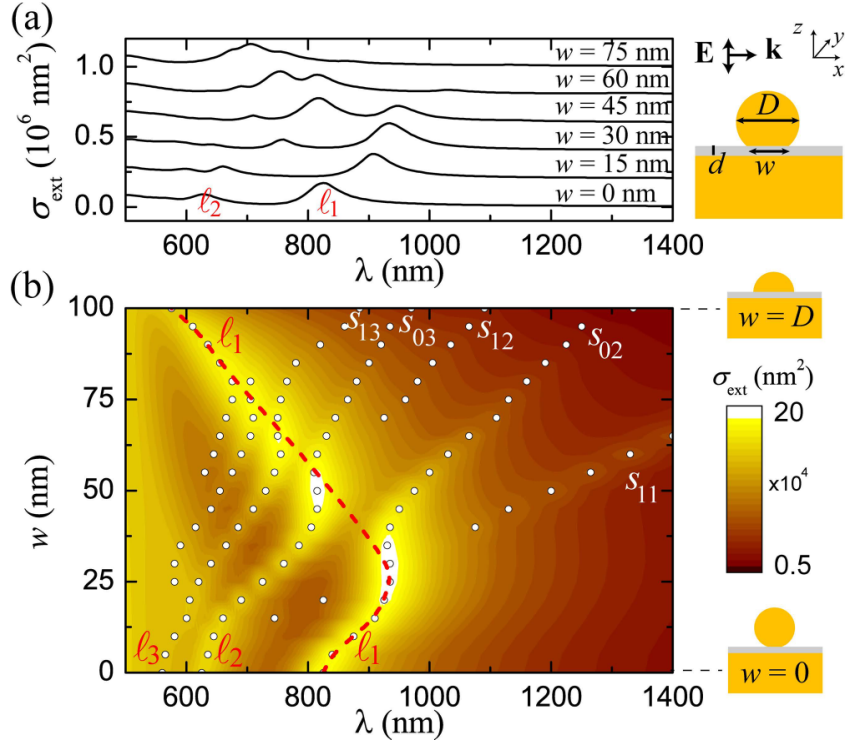

FIG. 1. (Color online) (a) Extinction spectra for faceted spherical gold NPs with diameter $D=100 \mathrm{~nm}$, separated from a 70-nm-thick gold film by a dielectric spacer of thickness $d=0.6 \mathrm{~nm}$ and permittivity $\varepsilon_{\mathrm{d}}=1.63$, as in the schematics to the right. Consecutive curves correspond to NPs of increasing circular facet diameter $w$ from bottom $(w=0 \mathrm{~nm})$ to top $(w=75 \mathrm{~nm})$ in steps of $15 \mathrm{~nm}$. The spectra are vertically shifted for clarity. (b) Mapped extinction spectra of the NPoM geometry described in (a) as a function of $w$ (from perfectly spherical NP to hemispherical NP as in the schematics to the right). Open circles trace the resonance peaks of all excited modes. Two different sets of modes can be identified and are labeled as $\ell$ and $s$ modes, according to their symmetry. The red dashed line is a guide to the eye of the $\ell_{1}$ mode.

When a perfectly spherical metallic NP is placed on a metallic film in a NPoM geometry, a localized plasmon is produced at the gap, formed by the interaction of the particle with its mirror image in the film, similar to the situation in a plasmonic NP dimer $[15,16,30]$. This antenna bonding dimer plasmon mode $\ell_{1}$ dominates the far-field spectra for illumination polarized along the NP-substrate axis. For very small separations, higher-order antenna modes, $\ell_{2}, \ell_{3}, \ldots$, are also efficiently excited [16,31]. The excitation of these antenna modes can be observed in the extinction spectrum of Fig. 1(a) (lowest spectrum, $w=0 \mathrm{~nm}$ ) and in the lowest part of the extinction contour map of Fig. 1(b), showing a dipolar $\ell_{1}$ mode at wavelength $\lambda=825 \mathrm{~nm}$ and a quadrupolar $\ell_{2}$ mode at $\lambda=625 \mathrm{~nm}$.

We now modify the spherical shape of the NP above the gap by introducing circular facets at the particle base. The facets are produced by cutting spherical caps off the bottom of the nanosphere, thus reducing its total height. In Fig. 1(a), we show the extinction spectra for increasing facet widths, from 15 to $75 \mathrm{~nm}$, in steps of $15 \mathrm{~nm}$. Introduction of the facets increases the interaction between the NP and the metal film due to the larger area interface. This leads to an initial strong redshift of the antenna bonding modes for small facets, as seen when comparing the $w=0,15$, and $30 \mathrm{~nm}$ cases in Fig. 1(a), and more clearly in Fig. 1(b). A higher-order antenna mode $\left(\ell_{3}\right)$ can also be excited at shorter wavelength. At larger facet diameters $(>25 \mathrm{~nm})$, this redshift saturates, and the modes eventually begin to blueshift, as the total NP height is reduced. This is particularly clear for the first antenna mode $\ell_{1}$, whose evolution with $w$ is marked in Fig. 1(b) by a red dashed line. We note here that the corresponding blueshift for perfectly spherical NPs under the same height reduction (through reduction of the NP diameter) is much smaller, as verified by detailed simulations, indicating that NP faceting further influences the modal shifts. Finally, by further increasing the facet width, a very rich structure of modes is clearly observed [upper part of Fig. 1(b)]. Most notably, additional weaker resonances are observed in the extinction spectra [marked by circles in Fig. 1(b) for clarity] away from the bright antenna modes [whose resonance peaks are also marked by circles in Fig. 1(b)], with an almost linear dependence on $w$. We denote these modes as $s_{m n}$, for symmetry reasons that will become clear below. These weak resonances are directly related to the interaction between the flat metal surfaces at the bottom of the particle and the substrate, in a metal-insulator-metal (MIM) cavity configuration [18]. Interestingly, $\ell$ and $s$ modes can either cross without interaction, as for example in the case of the $\ell_{1}$ and $s_{11}$ modes in Fig. 1(b), or they can interact, leading to a mode anticrossing and the opening of a wavelength gap, as is most strikingly observed for $\ell_{1}$ and $s_{02}$. Understanding the symmetry rules that govern this complex structure of NPoM modes and their interactions is the main aim of this paper.

In order to reveal the nature of the two different sets of $\ell$ and $s$ modes, we analyze in Fig. 2 the far-field spectra for two different cases with small [Fig. 2(a)] and large [Fig. 2(b)] facet widths. Two different morphological situations at the gap are thus obtained. Electric field enhancement $\left(E / E_{0}\right)$ contours of the key resonances at the gap midplane ( $x y$ plane parallel to the substrate) are also shown in the figure. For a small facet $w=15 \mathrm{~nm}$ [see [i] in Fig. 2(a)], the extinction spectrum [identical to that shown at the bottom of Fig. 1(a)] shows three dominant modes, $\ell_{1-3}$, whose bonding character is verified by their corresponding near-field maps. These modes exhibit large field enhancement at the top of the particle (not shown), forming antenna modes that are strongly radiative. At the same time, their electric fields are also strongly localized within the gap, with nodes that increase in number with mode order [see [ii] in Fig. 2(a)] [18]. We note that the field profiles of the $\ell_{2}$ and $\ell_{3}$ modes are not perfect concentric circles, mainly due to the slight mixing with the low-wavelength branches of the cavity $s_{m n}$ modes [see Fig. 1(b)].

For large facets, a totally different far- and near-field optical response can be observed, as shown in Fig. 2(b) for $w=$ $75 \mathrm{~nm}$. We first analyze the lowest-energy modes which are weakly radiative or nonradiative and are labeled as $s_{11}(\lambda=$ $1525 \mathrm{~nm}), s_{02}(\lambda=1130 \mathrm{~nm}), s_{12}(\lambda=960 \mathrm{~nm})$, and $s_{03}(\lambda=$ $865 \mathrm{~nm}$ ). The remaining nonradiative $s$ modes are higher-order resonances that interact with the broad $\ell_{1}$ antenna mode. Two of the modes, $s_{11}$ and $s_{12}$, appear only as resonance peaks in absorption but not scattering [see also enlarged view in inset of Fig. 2(b)], while the $s_{02}$ and $s_{03}$ modes produce small scattering resonances as well. All of these peaks are accompanied by very strong near-field enhancement in the gap, as shown in the field maps of [i] in Fig. 2(b). We identify the weakly radiating $s$ modes as transverse cavity resonances whose local field profiles are very similar to those produced in optical 
(a) [1]

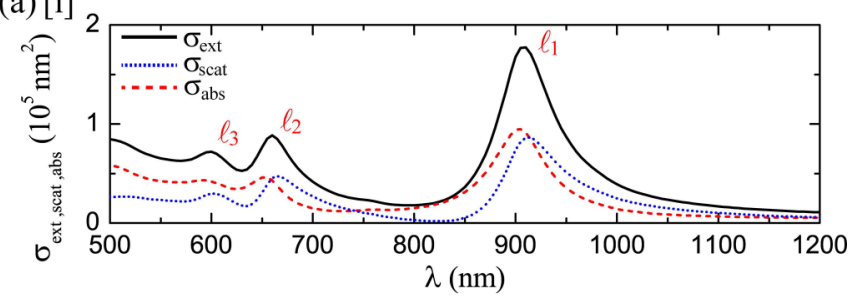

[ii]

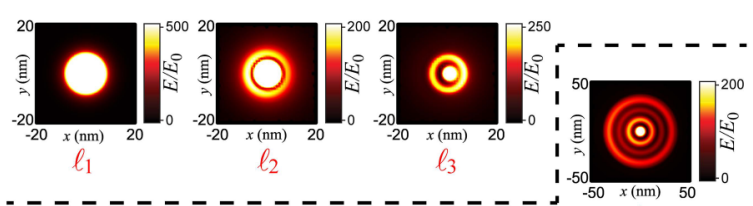

(b) $[\mathrm{i}]$
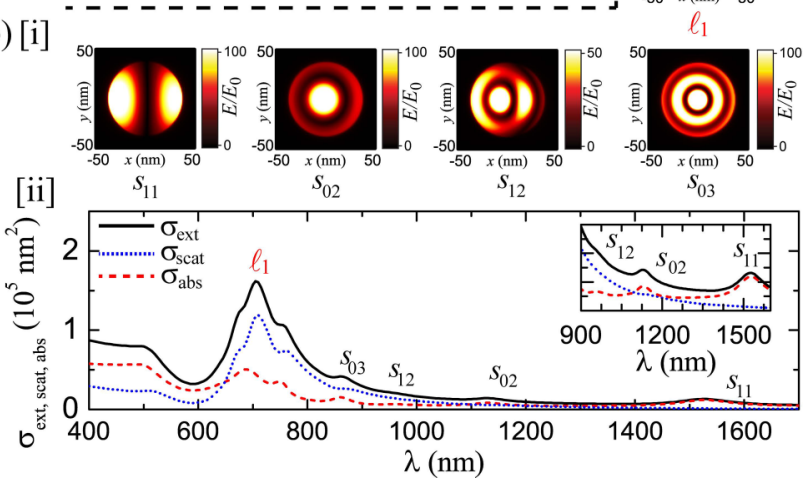

FIG. 2. (Color online) (a) [i] Extinction (black solid line), scattering (blue dotted line), and absorption (red dashed line) spectra for the NPoM geometry described in Fig. $1(w=15 \mathrm{~nm})$. [ii] Electric field enhancement $E / E_{0}$ in the $x y$ plane parallel to the substrate across the middle of the gap, for the three antenna resonances identified in [i]: $\ell_{1}(\lambda=910 \mathrm{~nm}), \ell_{2}(\lambda=660 \mathrm{~nm})$, and $\ell_{3}(\lambda=600 \mathrm{~nm})$. (b) Same as (a) for facet width $w=75 \mathrm{~nm}$. [i] Local field maps corresponding to the resonances identified in [ii] as $s_{11}(\lambda=1525 \mathrm{~nm}), s_{02}(\lambda=$ $1130 \mathrm{~nm}), s_{12}(\lambda=960 \mathrm{~nm}), s_{03}(\lambda=865 \mathrm{~nm})$, and $\ell_{1}(\lambda=705 \mathrm{~nm})$. [ii] Extinction, scattering, and absorption spectra, enlarged in the inset for longer wavelengths to show the first three $s$ modes.

patch antennas sustaining MIM modes [32-34]. To support this claim, we compare their dispersion with that obtained for the modes of the equivalent MIM cavity.

In the limit of very thin gaps of thickness $d$, the dispersion of the MIM modes is given by [35]

$$
\left(\frac{k}{k_{0}}\right)^{2}=\varepsilon_{\mathrm{d}}+\frac{\gamma}{2}\left[1+\sqrt{1+4\left(\varepsilon_{\mathrm{d}}-\varepsilon_{\mathrm{m}}\right) / \gamma}\right],
$$

where $k_{0}=2 \pi / \lambda$ is the wave number in vacuum, $k$ is the corresponding wave number in the MIM cavity, and $\gamma=$ $\left[\left(2 \varepsilon_{\mathrm{d}}\right) /\left(k_{0} d \varepsilon_{\mathrm{m}}\right)\right]^{2}$. From this dispersion, the effective refractive index of the cavity can be obtained as $n_{\text {eff }}=\operatorname{Re}(k) / k_{0}$. This refractive index can then be used to match the Fabry-Pérot condition for transverse magnetic wave resonances in our finite-size cylindrical gap of width $w[32,36]$,

$$
\lambda_{m n}=\frac{\pi w n_{\mathrm{eff}}}{a_{m n}-\beta},
$$

where $a_{m n}$ is the $n$th root of the $m$ th-order Bessel function $J_{m}$, with $m$ associated here with the azimuthal dependence of the modes in the cylindrical symmetry and $\beta$ an appropriate reflection phase $[32,37]$. Here we use $\beta=\pi / 2$, as we find that

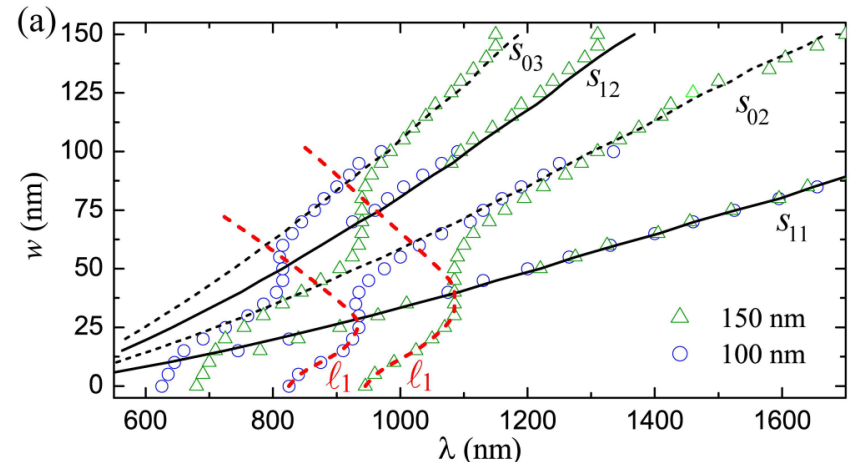

(b)

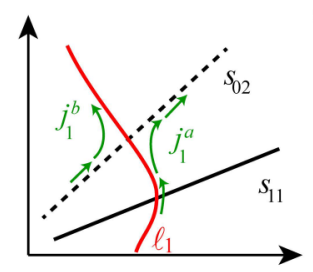

(c)

FIG. 3. (Color online) (a) Resonance peaks for $100 \mathrm{~nm}$ (blue circles) and $150 \mathrm{~nm}$ (green triangles) faceted gold nanospheres in the NPoM geometry as a function of facet diameter $w$. Black solid and dashed lines correspond to the $s_{1 n}$ and $s_{0 n}$ modes of the MIM cavity model in Eq. (2), respectively. The red dashed lines follow the evolution of the $\ell_{1}$ antenna modes for the two NP sizes. (b) Schematic interactions between the first antenna $\left(\ell_{1}\right)$ and cavity $\left(s_{11}\right.$ and $s_{02}$ ) modes shown in (a), with the hybrid $j_{1}^{a, b}$ modes following the green arrows and a hybridization gap opening between them. (c) Charge distribution inside the cavity for the $\ell_{1}, s_{11}$, and $s_{02}$ modes.

the field shows a maximum near the edge of the cavity instead of a node. A set of $w$-dependent modes, whose resonance wavelengths are shown in Fig. 3(a) by solid ( $m=1)$ or dashed $(m=0)$ black lines, is obtained from Eq. (2).

The MIM cavity resonances obtained from Eqs. (1) and (2) are compared in Fig. 3(a) with the resonances obtained from our simulations in the NPoM geometry for two different NP sizes, $D=100 \mathrm{~nm}$ (blue circles) and $D=150 \mathrm{~nm}$ (green triangles). The evolution of the antenna modes (only $\ell_{1}$ and $\ell_{2}$ are shown in the figure) with facet width is observed to depend strongly on NP diameter (green versus blue symbols). The cavity modes, on the other hand, do not depend on NP size but only on the diameter of the facet; therefore, any particle size tends to the same asymptotic behavior for increasing facet width. The results of Eq. (2) very accurately reproduce all of the $s$ modes obtained in the simulations throughout the entire facet width range. This justifies their labeling as $s_{m n}$, as the $n$th root of the corresponding $m$-order Bessel function.

In order to further investigate the dependence of antenna and cavity modes on the parameters defining the NPoM geometry system, namely NP size and spacer thickness, we show in Fig. 4 the extinction cross section of a NPoM similar to the one in Fig. 1(b), for a slightly smaller NP diameter $(D=80 \mathrm{~nm})$. We focus on the spectral range where the crossing between the $\ell_{1}$ antenna mode and the first two cavity modes, $s_{11}$ and $s_{02}$, occurs. In Fig. 4(a), we assume a fixed spacer thickness $d$ equal to $0.6 \mathrm{~nm}$ [similar to the one in Fig. 1(b)]. Consistent with the results found in Fig. 3(a), where the spectral differences were studied for NPs of different sizes, we find that while the antenna 


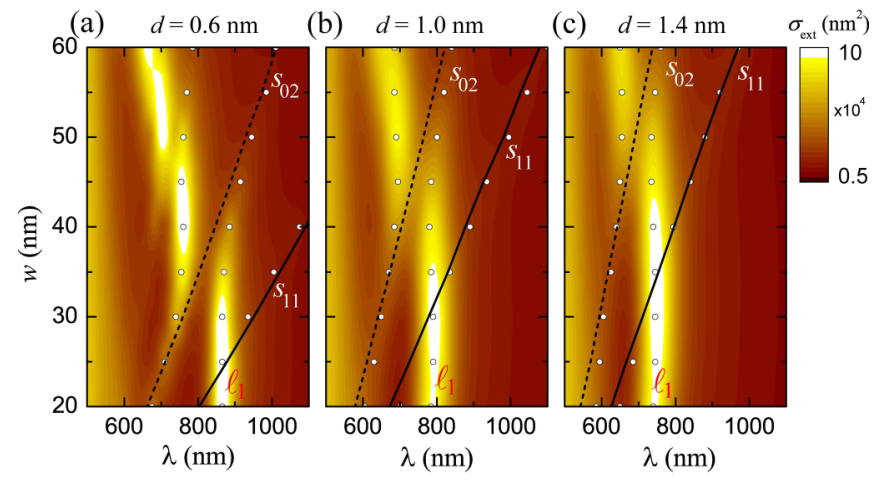

FIG. 4. (Color online) (a) Extinction cross section $\sigma_{\text {ext }}$ for a faceted spherical gold NP in the NPoM geometry, where the NP diameter is $D=80 \mathrm{~nm}$ and the spacer thickness $d=0.6 \mathrm{~nm}$. The spectral range where the first two cavity modes, $s_{11}$ and $s_{02}$, cross the $\ell_{1}$ antenna mode is shown. Open circles trace the resonance peaks of each mode. Black solid and dashed lines correspond to the $s_{11}$ and $s_{02}$ modes of the MIM cavity model in Eq. (2), respectively. (b), (c) Same as in (a), for spacer thickness separation $d=1.0 \mathrm{~nm}$ and $d=1.4 \mathrm{~nm}$, respectively.

modes show a strong dependence on NP size, the cavity modes are not affected by the choice of NP size [Fig. 1(b) vs Fig. 4(a)], but rather only depend on the facet width $w$, as derived from Eq. (2). The nanoparticle size is thus not a relevant parameter in influencing the cavity modes. On the other hand, the separation between the NP and the substrate, given by the spacer thickness $d$, strongly affects both antenna and cavity modes. As can be observed in Figs. 4(a)-4(c), the spectral position of the longitudinal antenna modes [bright features in the contour plots of Figs. 4(a)-4(c)] as well as the cavity modes [black lines in Figs. 4(a)-4(c)] shifts strongly when increasing the spacer thickness from $d=0.6 \mathrm{~nm}$ [Fig. 4(a)] to $d=1.0 \mathrm{~nm}$ [Fig. 4(b)] and $d=1.4 \mathrm{~nm}$ [Fig. 4(c)]. As $d$ increases, the interaction between the NP and the underlying metallic film is reduced, leading to blueshifts of the antenna modes. A similar effect is produced in the cavity modes, which are blueshifted when increasing $d$, due to a smaller confinement that produces a weaker field enhancement inside the cavity. These shifts are excellently tracked by Eq. (2), as shown by the solid and dashed lines in the three panels of Fig. 4 for the $s_{11}$ and $s_{02}$ modes, respectively. Furthermore, Eq. (2) predicts that for spacer thickness of $\approx 5 \mathrm{~nm}$, the facet width required for efficient excitation of the $s_{02}$ mode is larger that the NP diameter, thus preventing the possibility for such cavity modes to be sustained in well-separated NPoM geometries. The trends in Fig. 4 thus show how the systematic and predictive structure of the NPoM modes evolves in terms of the main parameters involved in the NPoM configuration, namely, particle diameter, facet width, and separation thickness.

\section{ANTENNA-CAVITY MODE HYBRIDIZATION}

A remarkable aspect of the dispersion curves in Fig. 3(a) is that the antenna modes cross the cavity modes with oddorder Bessel function $\left(s_{11}, s_{12}, \ldots\right)$ whereas they anticross the even orders $\left(s_{02}, s_{03}, \ldots\right)$, leading to the formation of hybrid modes. The different hybrid cavity modes $j_{1,2, \ldots}$ originate from antenna bonding longitudinal modes $l_{1,2, \ldots}$. The evolution of crossings and anticrossings is shown schematically in Figs. 3(b) and 3(c) in terms of mode symmetry and charge distribution, for the specific example of modes $\ell_{1}, s_{11}$, and $s_{02}$. The $s_{1 n}$ modes (azimuthal number $m=1$ ) cross the $\ell$ modes without interaction because they possess different symmetry. While the $m=1$ cavity modes exhibit a transverse dipolar nature and swap surface charge sign at each end of the gap, the $\ell$ modes are radially symmetric. Therefore, these two sets of orthogonal modes cannot couple and they cross unperturbed. On the other hand, the $s_{0 n}$ modes (azimuthal number $m=0$ ) share the same symmetry as the $\ell$ modes. When both modes approach spectrally, they interact and strongly anticross, forming hybrid $j_{n}$ modes, and thus opening a hybridization gap. The $s_{01}$ mode is not excited since its charge distribution, though compatible in symmetry with the $\ell_{1}$ mode, would produce net charge in the particle.

To show how the interaction between the $\ell$ and $s$ modes depends on the exact morphological details of the cavity and, more specifically, on the opening of the facet edges that determine the degree of coupling of both sets of modes, we show in Fig. 5(a) the width of the resulting hybridization gap for five different NP facet edge terminations. To obtain this, we calculate extinction maps similar to the one shown in Fig. 1(b). In these maps, for each $w$ the corresponding NP height is always the same, regardless of NP shape, but the curvature of its sides is reduced, thus shifting the geometry from a sphere to an ellipsoid and finally to a nanorod (with right-angle facet corners), as schematically depicted on the right-hand side of Fig. 5(a). We then determine from each extinction map the minimum spectral gap width, and the facet width $w$ for which this minimum occurs. For this $w$, we define the angle $\alpha$ between the sidewall and the facet bottom face, as shown in the inset of Fig. 5(a). This angle $\alpha$ gives a measure of the geometrical projection of the particle termination on the substrate and determines the strength of the coupling for each $j$ mode. The width of the gap obtained for each case is shown by colored dots in Fig. 5(a). As the sidewall angle at the particle edge is increased and the sides of the particle become more steep, eventually tending to a straight $\operatorname{rod}\left(\alpha \rightarrow 90^{\circ}\right)$, the interaction between the $\ell$ and $s$ modes reduces, closing the spectral anticrossing of the hybrid modes. This latter situation is similar to the case of flat dimer antennas, where no anticrossing of the antenna $\ell_{1}$ mode and the cavity modes is found [39]. The dependence of coupling between antenna and cavity modes on the fine details of the gap morphology (facet size and angle of termination) thus provides a valuable optical tool to track atomic-scale modifications in gap formation and faceting, involving material migration or photochemical modifications [18].

Finally, to better display the mechanisms governing the interaction between antenna and cavity modes, we discuss how the original uncoupled $\ell$ and $s$ modes can be retrieved from the hybrid $j$ modes by simply adding and subtracting the two branches of the electric field distributions of $j$ on either side of the hybridization gap. In this procedure, an appropriate weighting factor $f$, which takes into account the relative strengths of the two uncoupled modes, is introduced. This decomposition factor clearly depends on the angle at the edge of the particle, $\alpha$, although a simple expression showing 
(a)

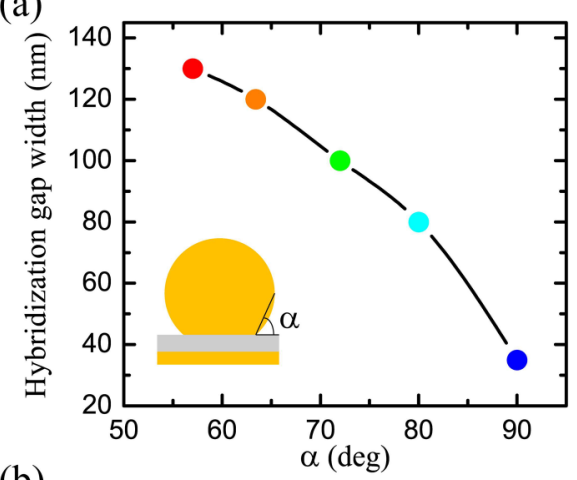

(b)

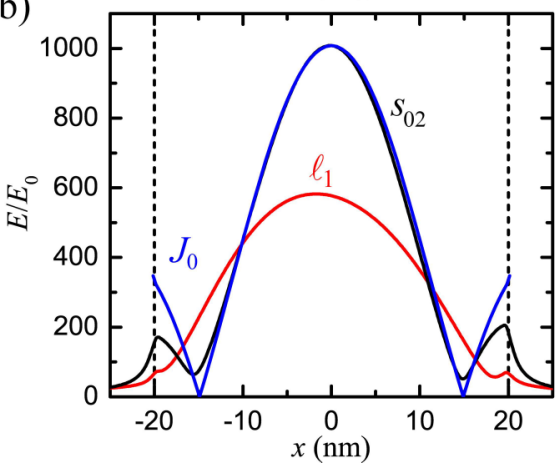

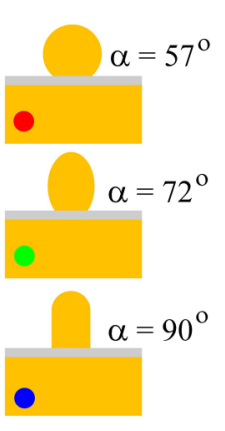

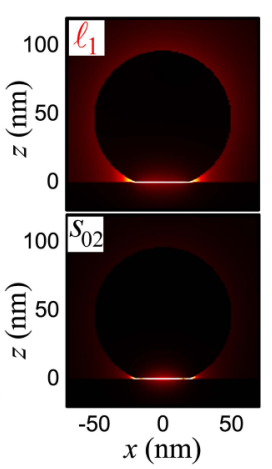

FIG. 5. (Color online) (a) Interaction between the $\ell_{1}$ and $s_{02}$ modes, expressed in terms of the width of the produced hybridization gap, as a function of the angle $\alpha$ between the nanoparticle sidewall and the facet bottom face (see inset). Colored dots for the different angles map directly to the corresponding right-hand schematics. (b) Normalized electric field amplitude of the decomposed $\ell_{1}$ (red line) and $s_{02}$ (black line) modes for a spherical NP $\left(\alpha=57^{\circ}\right)$, along the middle of the cavity, together with the distribution of the normalized $J_{0}$ Bessel function along the cavity (blue line). Vertical dashed lines denote the facet edges of the plasmonic cavity. Electric field distributions of the two modes around the NP are shown on the right. Both contours are plotted with the same color map, saturated at a maximum field enhancement $E / E_{0}=50$.

this dependence is not straightforward. In the following, we use estimated $f$ values so that the field distributions for the resulting uncoupled modes are similar to those obtained numerically for the corresponding modes far from hybridization, both inside the cavity and around the NP. Such a decomposition is shown in Fig. 5(b) for the $\ell_{1}$ and $s_{02}$ modes in the case of a spherical NP $\left(\alpha=57^{\circ}\right)$ with $w=40 \mathrm{~nm}$. The decomposed modes are obtained from the hybrid $j_{1}^{a}(\lambda=935 \mathrm{~nm})$ and $j_{1}^{b}$ $(\lambda=805 \mathrm{~nm})$ modes [see Fig. 1(b)] through $\left(\begin{array}{l}\ell_{s_{0}} \\ )\end{array}\right)=j_{1}^{a} \pm f j_{1}^{b}$, where $f=0.35$. The obtained electric field profiles across the middle of the cavity are displayed on the right-hand side of Fig. 5(b) for the $\ell_{1}$ (red line) and $s_{02}$ (black line) uncoupled modes. The normalized Bessel $J_{0}$ function (blue line) matches the shape of the decomposed $s_{02}$ mode very well. From the field distributions, a clear radiative nature can be observed for the $\ell_{1}$ mode (upper field map), while a strong field localization inside the cavity (with a radial node) and negligible field

enhancement around the rest of the particle is observed for the $s_{02}$ mode (lower field map), as expected by corresponding field profiles away from the anticrossing. An estimate of the interaction between these two modes can be obtained by calculating the Coulomb coupling between the charge distributions, $\sigma_{\ell}$ and $\sigma_{s}$, of the uncoupled modes at the cavity, $\ell$ and $s$, proportional to $\iint \sigma_{\ell}(\boldsymbol{r}) \sigma_{s}\left(\boldsymbol{r}^{\prime}\right) d \boldsymbol{S} d \boldsymbol{S}^{\prime} /\left|\boldsymbol{r}-\boldsymbol{r}^{\prime}\right|$ [38]. This interaction framework can also further elucidate why the $s_{1 n}$ modes do not couple with the $\ell$ modes: the two modes of different azimuthal symmetry give a zero-valued interaction integral over the entire cavity surface. The evolution of the normalized mode splitting (interaction energy) as a function of $\alpha$ obtained from this estimation shows very good agreement with the simulations of Fig. 5(a) for every NP morphology, although the results are found to depend strongly on the selection of $f$. We should note that the decomposition procedure described above is not strict and further work, considering for example mode normalization, is required to establish a rigorous decomposition method. However, it is useful to show a simple and intuitive picture, as presented here, to understand the nature of interactions between the two sets of modes.

\section{CONCLUSION}

In summary, we have studied the rich optical response of plasmonic nanogaps in the NPoM geometry, which forms an increasingly important configuration in molecular sensing, photochemistry, and optoelectronics. As nano-optics now probes atomic-scale processes by means of singular spectral fingerprints, the dependence of plasmonic modes on the morphology and symmetries becomes crucially important. We identify the set of hybrid plasmonic modes in faceted nanogaps that are the product of mixing longitudinal antenna $\ell$ modes and transverse cavity $s$ modes, loosely analogous to spin-orbit mixing in atomic Stark ladders. The evolution of these modes depends on the symmetry and morphology of the nanogap, producing crossings and anticrossings tunable on demand. An understanding of the properties of these NPoM modes is thus important for engineering novel plasmonic nanostructures with complex, controllable response in the visible and near-infrared range.

\section{ACKNOWLEDGMENTS}

C.T., R.E., and J.A. acknowledge financial support from Project No. FIS2013-41184-P of MINECO, and Project No. Etortek 2014-15 of the Department of Industry of the Basque Government. R.E. also acknowledges support from the program Fellows Gipuzkoa from the Gipuzkoako Foru Aldundia through FEDER funds of the European union "Una manera de hacer Europa." J.J.B. acknowledges financial support from EPSRC Grants No. EP/G060649/1, No. EP/L027151/1, and No. EP/K028510/1, ERC Grant No. LINASS 320503, and Ikerbasque. J.M. acknowledges support from the Winton Programme for the Physics of Sustainability.
[1] E. Prodan, C. Radloff, N. J. Halas, and P. Nordlander, Science 302, 419 (2003).
[2] P. Nordlander, C. Oubre, E. Prodan, K. Li, and M. I. Stockman, Nano Lett. 4, 899 (2004). 
[3] H. Xu, J. Aizpurua, M. Käll, and P. Apell, Phys. Rev. E 62, 4318 (2000).

[4] E. Hao and G. C. Schatz, J. Chem. Phys. 120, 357 (2004).

[5] H. Chen, G. C. Schatz, and M. A. Ratner, Rep. Prog. Phys. 75, 096402 (2012).

[6] A. Kinkhabwala, Z. Yu, S. Fan, Y. Avlasevich, K. Müllen, and W. E. Moerner, Nat. Photon. 3, 654 (2009).

[7] X. Zhang, Y. L. Chen, R.-S. Liu, and D. P. Tsai, Rep. Prog. Phys. 76, 046401 (2013).

[8] H. Choo, M.-K. Kim, M. Staffaroni, T. J. Seok, J. Bokor, S. Cabrini, P. J. Schuck, M. C. Wu, and E. Yablonovitch, Nat. Photon. 6, 838 (2012).

[9] A. P. Alivisatos, K. P. Johnsson, X. Peng, T. E. Wilson, C. J. Loweth, M. P. Bruchez Jr., and P. G. Shultz, Nature (London) 382, 609 (1996).

[10] S. J. Barrow, A. M. Funston, D. E. Gómez, T. J. Davis, and P. Mulvaney, Nano Lett. 11, 4180 (2011).

[11] R. W. Taylor, T.-C. Lee, O. A. Scherman, R. Esteban, J. Aizpurua, F. M. Huang, J. J. Baumberg, and S. Mahajan, ACS Nano 5, 3878 (2011).

[12] J. Kern, S. Gromann, N. V. Tarakina, T. Häckel, M. Emmerling, M. Kamp, J-S. Huang, P. Biagioni, J. C. Prangsma, and B. Hecht, Nano Lett. 12, 5504 (2012).

[13] P. K. Jain and M. A. El-Sayed, Chem. Phys. Lett. 487, 153 (2010).

[14] H. Duan, A. I. Fernández-Domínguez, M. Bosman, S. A. Maier, and J. K. W. Yang, Nano Lett. 12, 1683 (2012).

[15] C. Ciracì, R. T. Hill, J. J. Mock, Y. Urzhumov, A. I. FernándezDomínguez, S. A. Maier, J. B. Pendry, A. Chilkoti, and D. R. Smith, Science 337, 1072 (2012).

[16] J. Mertens, A. L. Eiden, D. O. Sigle, F. Huang, A. Lombardo, Z. Sun, R. S. Sundaram, A. Colli, C. Tserkezis, J. Aizpurua, S. Milana, A. C. Ferrari, and J. J. Baumberg, Nano Lett. 13, 5033 (2013).

[17] J. B. Lassiter, F. McGuire, J. J. Mock, C. Ciracì, R. T. Hill, B. J. Wiley, A. Chilkoti, and D. R. Smith, Nano Lett. 13, 5866 (2013).

[18] D. O. Sigle, J. Mertens, L. O. Herrmann, R. W. Bowman, S. Ithurria, B. Dubertret, Y. Shi, H. Y. Yang, C. Tserkezis, J. Aizpurua, and J. J. Baumberg, ACS Nano 9, 825 (2015).
[19] B. P. Joshi and Q.-H. Wei, Opt. Express 16, 10315 (2008).

[20] J. Yang, C. Sauvan, A. Jouanin, S. Collin, J.-L. Pelouard, and P. Lalanne, Opt. Express 20, 16880 (2012).

[21] M. Kuttge, F. J. García de Abajo, and A. Polman, Nano Lett. 10, 1537 (2010).

[22] K. J. Savage, M. M. Hawkeye, R. Esteban, A. G. Borisov, J. Aizpurua, and J. J. Baumberg, Nature (London) 491, 574 (2012).

[23] J. A. Scholl, A. García-Etxarri, A. L. Koh, and J. A. Dionne, Nano Lett. 13, 564 (2013).

[24] T. V. Teperik, P. Nordlander, J. Aizpurua, and A. G. Borisov, Phys. Rev. Lett. 110, 263901 (2013).

[25] Y. Luo, A. I. Fernandez-Dominguez, A. Wiener, S. A. Maier, and J. B. Pendry, Phys. Rev. Lett. 111, 093901 (2013).

[26] P. B. Johnson and R. W. Christy, Phys. Rev. B 6, 4370 (1972).

[27] A. Molina-Sánchez and L. Wirtz, Phys. Rev. B 84, 155413 (2011).

[28] F. J. García de Abajo and J. Aizpurua, Phys. Rev. B 56, 15873 (1997).

[29] F. J. García de Abajo and A. Howie, Phys. Rev. B 65, 115418 (2002).

[30] N. Yamamoto, S. Ohtani, and F. J. García de Abajo, Nano Lett. 11, 91 (2011).

[31] I. Romero, J. Aizpurua, G. W. Bryant, and F. J. Carcía de Abajo, Opt. Express 14, 9988 (2006).

[32] R. Filter, J. Qi, C. Rockstuhl, and F. Lederer, Phys. Rev. B 85, 125429 (2012).

[33] R. Esteban, T. V. Teperik, and J. J. Greffet, Phys. Rev. Lett. 104, 026802 (2010).

[34] F. Minkowski, F. Wang, A. Chakrabarty, and Q.-H. Wei, Appl. Phys. Lett. 104, 021111 (2014).

[35] S. I. Bozhevolnyi and T. Sondergaard, Opt. Express 15, 10869 (2007).

[36] J. D. Jackson, Classical Electrodynamics, 3rd ed. (Wiley, New York, 1999).

[37] R. Gordon, Phys. Rev. B 73, 153405 (2006).

[38] E. Prodan and P. Nordlander, J. Chem. Phys. 120, 5444 (2004).

[39] R. Esteban, G. Aguirregabiria, A. G. Borisov, Y. M. Wang, P. Nordlander, G. W. Bryant, and J. Aizpurua, ACS Photon. 2, 295 (2015). 